\title{
A model for the study and design of teaching situations with ICT
}

\author{
Steve Kennewell, Howard Tanner and John Parkinson \\ Department of Education, University of Wales Swansea, Swansea SA2 7NB, UK. \\ s.e.kennewell@swansea.ac.uk
}

Keywords: learning theory, affordance, pedagogy, evaluation

\begin{abstract}
Any situation in which activity takes place provides affordances for the activity. We use this idea first to characterise learning, and then to discuss the way in which the educational environment is manipulated by the effective teacher in order to foster learning. ICT provides some particular affordances which successful teachers exploit in their classroom practice, and the use of an affordance model helps us to analyse the pedagogy involved. We provide a number of practical illustrations and draw general conclusions concerning the effective use of ICT in teaching and learning.
\end{abstract}

\section{INTRODUCTION}

During the first 20 years of microcomputers in education, much has been achieved by enthusiastic teachers who have recognised the power of the medium of ICT in their teaching. As the medium becomes as pervasive in schools as it is in other walks of life, we expect its features to be harnessed by all teachers in order to help promote learning.

Mere exposure to a medium is not, however, any guarantee that learning will occur. Saloman (1997), while examining how various media influence the representation of information, points out that children perceive TV as a medium to be fun, simple, and useless. Consequently they learn nothing from it, unless they are told that learning is expected of them. This is in contrast to the early days of TV, when it was perceived as a serious medium, and much learning took place. 
As ICT becomes a natural feature of children's lives, we can observe that ICT, too, is seen as simple and fun. There are already signs (Robertson 1997) that ICT may have a similarly impoverished relationship to learning at the start of the 21 st century that TV has had towards the end of the 20th. Teachers must therefore manage the use instructional use of ICT carefully if it is to fulfil our clirrent expectations.

The importance of pedagogy in the study of ICT and learning has been highlighted in research (Somekh and Davies 1991; Kennewell 1997) and in UK government policy (TTA 1998). However, our ability to characterise the teacher's contribution to the ICT learning situation has been hampered by the lack of an adequate framework for analysis. Traditional models of instructional design and evaluation for ICT are based on rationalist assumptions about educational objectives and teacher planning, together with positivist ideas about the relationship between instructional material and individual cognition. Squires and MacDougall (1994) have highlighted the inadequacies of traditional models, and have developed instead the Perspectives Interaction Paradigm for evaluating educational software. Crook (1991) argues for the application of cultural psychology if we are to evaluate computer-based interventions effectively:

To be valuable, an evaluation must be sufficiently open-ended to unravel

what is being learned and where the learning is located. (1991:82)

We have refined these interpretivist ideas to create a framework which takes into account the whole of the environment in which the learner and the computer are situated - the teacher, the other resources, the other learners, and the cultural context, as well as the knowledge that the individual brings to the classroom.

We first explain some of the terminology and relationships that we have found useful, and then illustrate how they can be used to interpret some particular didactic situations.

\section{ACTIVITY SITUATIONS, AFFORDANCES AND ABILITIES}

An activity situation comprises a person, some activity involving the person, and the environment in which this activity takes place. When studying an educational activity situation, we can focus our analysis on the individual learner, on the situation as a whole, or on the interaction between the individual and the environment during an activity In this paper, we will take the interaction as the focus of analysis. 
We will thus consider the relationship between:

- the individual learners - their cognitive and metacognitive skills, their concepts, their attitudes, and their goals;

- the environment in which the individual is situated - the location; the resources available; the other people involved such as the teacher and classmates.

Teachers are in a special position in the classroom, of course. They have some choice concerning what role to adopt, what the relative roles of pupils will be, what resources should be made available, and even the location. Other students, too, have an important influence over an individual's learning, but we will not be able to focus on this factor here.

We assume that the cognitive contribution which an individual brings to an activity is not fixed; it is dependent upon the particular features of the environment in which the activity is situated. Greeno (1994) uses Gibson's (1986) idea of affordances for the properties of the environment, and suggests the term abilities for the individual's potential to act within the environment. An activity will be completed satisfactorily if the individual's abilities are sufficient in combination with the affordances of the situation to enable the desired outcomes to be achieved.

We find it helpful to extend the idea of affordance to cover intentional support in social situations: thus we include the informal notions of help and guidance, and the more formal neo-Vygotskyan ideas of scaffolding (Bruner 1986); assisted performance (Tharp and Gallimore 1988); and contingent support for learning (Wood and Wood 1996). Abilities are also related to more familiar ideas: those of knowledge, skills and understanding. They should not be identified with an immutable 'intelligence', however.

\section{LEARNING AND TEACHING}

It is beyond the scope of this paper to consider abilities and how they develop in detail, but we do need to consider how to characterise learning in this perspective. We consider that learning has taken place when an individual's abilities change in some way. We cannot determine directly whether someone has learned anything in a particular situation, but we can characterise the changes observed in their future activity in situations with similar or reduced affordances. Clearly, then, we are adopting a situative view of learning (Greeno and Moore 1993).

It is quite possible, of course, for an activity (whether successful or unsuccessful) to result in no learning at all. In educational contexts, however, we presume that there is some expectation that learning will take 
place, and that teachers devise classroom situations with the intention of promoting learning. We will call these didactic situations.

Teachers typically plan, implicitly or explicitly, to manipulate the didactic situation so that:

1. the affordances are initially insufficient to enable the learners to be successful in their activity on the basis of their current abilities;

2. the affordances can then be increased by the teacher to enable the learners to succeed - an information sheet, a calculator, simpler language, a question, a demonstration, an analogy, a brainstorm, a discussion group, pairing with a more able student;

3. learners' abilities will change as a result of this experience.

Thus, when a didactic situation involves computer use, we need to consider: what the computer affords the learner in carrying out activities; how the computer's affordances relate to other affordances of the didactic situation; how the affordances may be manipulated by the teacher so as to promote learning.

In England, the Teacher Training Agency (TTA 1998) requires newly qualifying teachers to know:

the features of IC $T$ which can be used ... to support teaching and learning in subjects, including:

- speed and automatic functions ...

- capacity and range ...

- provisionality...

- interactivity ...

In the following two sections, we shall examine some examples of didactic situations which we have recently observed. We consider:

- how the above features of ICT which afford certain aspects of activity in the typical classroom;

- how ICT relates to other affordances;

- how learning is promoted.

Our focus is on the teacher, students and computers in this analysis, although the school culture, the location, and other resources in the classroom all have some influence on the learning which takes place. 


\section{EXAMPLES OF ICT'S ROLE IN DIDACTIC SITUATIONS}

\subsection{Situation 1: Simulation}

Students studying Roman life are using a multimedia simulation programme. They have to play the role of a Roman citizen, carrying out a trade in order to earn money, making decisions what to buy in order to provide for their families, and moving around the town to find the relevant shops and services they need. They achieve these simulated actions by clicking on appropriate screen images.

ICT affords cues and prompts for their decision making - options represented visually on screen, oral explanations of matters through headphones, graphics of money available and costs of products. It affords planning and evaluation of progress in the task, through structuring their environment, displaying quantities visually, and limiting their decisions if they attempt an action which the rules of the simulation do not allow.

\subsection{Situation 2: Multimedia database}

Pupils are using a multimedia CD-ROM on world religions to answer questions on a printed worksheet concerning Judaism and Islam. The questions are structured so that the first few require simple factual answers, the next ones require explanations, and the final ones require comparative discussion.

The computer affords selection of information for the factual questions through hierarchical searching or keyword searching with Boolean logic. These techniques require some abilities in search strategies from the students, and little success will be gained from trying to look at all the information haphazardly.

\subsection{Situation 3: Desk top publishing}

A group of students are working individually on a class of designing a logo for a business which they have selected for a marketing simulation. In the same class, another group is working on the same class with manual equipment.

The computer affords:

- the drawing of a number of pre-defined shapes, line styles, shadings;

- the writing of text in a variety of sizes and coherent, accurate type faces;

- the deletion, movement, and copying of any particular feature of the drawing; 
- the reflection of part of the drawing in order to achieve symmetry.

It also affords the selection of these facilities by providing symbols on the screen and menu headings which just have to be clicked to implement a feature or to choose an operation. It affords errors, of course, but it also affords instant 'undoability'. Above all, then, it affords experimentation.

\subsection{Situation 4: Arithmetic skills tutor}

Some students with learning difficulties are using software designed to develop their arithmetic skills. They are presented with subtraction sums set out to be solved by a standard written algorithm.

The students are first provided with a calculation to work out. They type in an answer, and if it is considered correct by the software, they receive a reassuring message. More calculations are provided in a graded sequence which covers most common errors in the intended algorithm. When they go wrong, the computer prompts them to check whether their answers are reasonable and gives them a chance to correct their working. If they still go wrong, the computer increases the affordances for carrying out the algorithm correctly. It prompts to write their working figures in suitable places, and if they write in an incorrect figure, the computer displays a clear message about their mistake and prompts them to try again. When they do this correctly, the affordance is reduced again.

\subsection{Situation 5: Word processing}

A class learning German as a foreign language is producing tourist information, in the target language, concerning a particular town which they are using as a setting for role plays, comprehension exercises and narrative writing. Using ICT, the students work in pairs to type in their first thoughts on what to write and then they check that the language is correct: the computer affords continuous evaluation of the text by the authors.

This could be supplemented using a spelling and grammar checker, but the teacher has found that these tools afford acceptance of inappropriate suggestions. They often lead the students often to change a word which was spelt only slightly wrongly into a completely different word. Furthermore, the computer's comments on grammar are not helpful to students with only a limited range of constructions. So instead, the teacher checks all pupils' work at each stage of development so that he can provide carefully targeted affordances for correction.

The teacher is expecting students to use a wide range of vocabulary and grammatical construction because the text is provisional: the computer affords 'editability'. When the language is correct, the students design the 
layout, import pictures, adjust the font and size, and print their professionallooking brochure. The computer affords effective presentation.

\section{LEARNING WITH ICT}

In Situation 1, the students are encountering aspects of Roman life through their surrogate experience. The intention of the software author is that, in order to prosper in this virtual world, they should understand certain relationships between the Roman people and their environment. A successful route through the simulation may be devised through the trial and error involved in the initial exploration, but the intended learning is only likely to arise through strategic thinking and discussion. This is afforded in two ways which do not depend on the computer: first, students are required to work in pairs or threes, so that decisions have to be discussed and justified to others; secondly, the teacher intervenes to ask questions concerning what they have found out and what they plan to try. These questions can direct the learners very generally or quite specifically, depending on the minimum degree of affordance the teacher decides will stimulate learning.

In Situation 2, there are two aspects to the learning. The first one is the factual detail of the two religions, their similarities and differences, their origins and development. Basic facts are unlikely to be retained from the simple experience of retrieving them from the CD-ROM and writing them down: the affordance for access is too high. Comparison and explanation, on the other hand, may be afforded by the computer to students who would find such analysis impossible using traditional linear text.

The second aspect is the process of enquiry from a large database. The computer affords the narrowing down or expansion of a search by means of Boolean operations. The students are unlikely to learn this naturally, however, because they are already able to search visually through text. Since the computer affords this visual searching by displaying easily readable sections of text and providing a familiar navigation procedure, there is no need for them to expend the mental effort required to use the search features. The teacher, then, must provide initially the strategic thinking which affords effective use of search techniques.

In Situation 3, similarly, there are two aspects to the learning: design concepts and CAD software techniques. There is a complex interplay between the two. The design skills are learned more easily through the CAD software which provides much greater affordances for the drawing process than the manual techniques. But the CAD software is not trivial, and some drawing abilities are not easily developed merely by attempting to apply the 
software features to tasks. The teacher thus stops the students at carefully judged moments in order to demonstrate particular features and explains how to use them purposefully in design. This demonstration and explanation affords application of the features to subsequent tasks.

In Situation 4, the computer itself is programmed with a mechanism for adjusting affordances so as to set tasks just ahead of the student's abilities. Whilst this may aid the memorisation of specific arithmetic techniques, it is more difficult to explain how such a mechanism could handle conceptual development. This is a more complex matter for which the expert teacher has a rich, extensive repertoire of activities and affordances, well-tuned to the ways in which learners think.

In Situation 5, the students are learning a greater range of language because they do not feel limited to the words and constructions which they already know well. Their goals can be set at a higher level without leading to conspicuous failure, because the computer affords the editing of text and the teacher can talk in terms of developing their work rather than correcting their errors.

\section{CONCLUSION}

Our illustrations show how didactic decision making can be represented using the constructs of affordances and abilities. We have considered how ICT can afford aspects of student activity, and what affects this may have on learning. In order to promote effective learning, the teacher sets tasks which are beyond the students' current abilities, and uses affordances to narrow the gap to one which the students can bridge themselves with some 'mental effort' (Saloman 1997). The computer's value is in the range of affordances for activity it can provide; the teacher's role is to manipulate the activities and affordances in order to promote learning.

If the affordances which the computer provides are not carefully judged, they may actually restrict the development of the intended abilities. This happens in the following circumstances.

1. The computer makes the task trivial, so that no mental effort is required and no learning is likely to take place.

2. The students may not have the abilities required to operate the computer as intended. In this case, instead of support for an activity, we have the 'IT interference factor' (Birnbaum 1990), and again there may be little or no learning.

3. Learning does take place, but the abilities developed are dependent on access to the computer. If the aim is develop conceptual abilities which 
can be utilised in non-computer contexts, then the teacher will need to design further didactic situations in which the computer is not used.

Our framework for analysis and planning of didactic situations is based on teachers' familiar practices - creating learning environments, designing and setting tasks, and interacting with students to manage their learning. The framework is sufficiently powerful to help describe and explain the processes we observe in the classroom, sufficiently general to incorporate the special role which ICT has in the learning process, and sufficiently practical to help teachers plan effective lessons.

\section{REFERENCES}

Bruner, J. (1986) Actual minds, possible worlds. Cambridge: Harvard University Press.

Crook, C. (1991) Computers in the zone of proximal development: Implication for evaluation. Computers and Education 17(1) 81-91.

Gibson, J. J. (1986) The ecological approach to visual perception. Hillsdale, NJ: Lawrence Erlbaum Associates.

Greeno, J. (1994) Gibson's affordances. Psychological Review 101(1) 336-342.

Greeno, J. and Moore, J. (1993) Situativity and symbols: Response to Vera \& Simon. Cognitive Science 17, 49-59.

Kennewell, S. (1997). The integration of information technology into teachers' decisionmaking. In D. Passey and B. Samways (eds.) Information technology: Supporting change through teacher education. London: Chapman and Hall.

Robertson, J. (1998) Paradise lost: Children, multimedia and the myth of interactivity. Journal of Computer Assisted Learning 14(1) 31-39.

Saloman, G. (1997) Of mind and media. Phi Delta Kappan January 1997, 375-380.

Somekh, B. and Davies, R. (1991) Towards a pedagogy for information technology. Curriculum Journal 2(2) 153-168.

Squires, D. and MacDougall, A. (1994) Choosing and using educational software. London: Falmer Press.

Tharp, R. and Gallimore, R. (1988) Rousing minds to life. Cambridge: Cambridge University Press.

TTA (1998) The use of ICT in subject teaching: Expected outcomes for teachers. London: Teacher Training Agency.

Wood, D. and Wood, H. (1996) Contingency in teaching and learning. Learning and Instruction 6(4) 391-397.

\section{BIOGRAPHIES}

Steve Kennewell taught mathematics and computing in secondary schools, then worked as a specialist computing teacher and IT Advisory teacher. Since 1990, he has been at the University of Wales Swansea. He teaches initial teacher training courses in mathematics and in IT as a subject, and Masters' courses in teaching and learning. His research interests include the development of IT capability and IT in mathematics education. He is joint editor of the journal Computer Education. 
John Parkinson taught chemistry in secondary schools in England and Wales for eight years before joining the Education Department at the University of Wales Swansea. He presently teaches on the Postgraduate Certificate in Education and Masters' degree courses and is responsible for supervising $\mathrm{PhD}$ students. His main areas of research include the study of pupils' attitudes towards science and ICT, and the development of ICT across the curriculum.

Howard Tanner taught Mathematics and IT in secondary schools for sixteen years before joining the Education Department in the University of Wales Swansea. He has been involved in several nationally funded research projects concerning the teaching and learning of mathematics and the development of IT capability. He is particularly interested in the impact of ICT on styles of teaching and learning. 